\title{
EVALUATION OF STAINING METHODS FOR DIAGNOSIS OF TRICHOMONIASIS IN CLINICALLY SUSPECTED WOMEN IN JAZAN, KSA.
}

By

HANAN Z. RAYAN ${ }^{1,2}$, WAFAA M. ZAKI ${ }^{1,2^{*}}$ AND AYMEN M. MADKHALI ${ }^{2}$

Department of Medical Parasitology, Faculty of Medicine, Suez Canal University, Ismailia, Egypt ${ }^{1}$, and Department of Medical Laboratory Technology, Faculty of Applied Medical Sciences, Jazan University, the Kingdom of Saudi Arabia ${ }^{2}$

( ${ }^{*}$ Correspondence: wafaa_zaki@hotmail.com)

\section{Abstract}

Forty female patients experiencing symptoms suggestive of trichomoniasis were included into the present investigation. Patients` self-obtained vaginal swabs were gathered and examined for $T$. vaginalis by wet mount, Giemsa stain, Gram stain and culture techniques. T. vaginalis was recognized in 11 out of 40 specimens by culture (27.5\%), 10 by wet mount (25\%), 8 by Gram stain (20\%) and 7 by Giemsa stain $(17.5 \%)$. Contrasted with culture as the standard technique, Giemsa staining method indicated $63.6 \%$ sensitivity compared with $54.5 \%$ for wet mount or Gram staining. Giemsa staining specificity was $100 \%$ contrasted with $86.2 \%$ for wet mount and $93.1 \%$ for Gram stain. In addition, a considerable agreement was found amongst culture and Giemsa staining $(\mathrm{Kappa}=0.717)$ while moderate agreement of Gram stain $($ Kappa $=0.52)$ and wet mount $($ Kappa $=0.419)$ with culture was found. Key words: Kingdom of Saudi Arabia, Trichomoniasis, Females, Diagnosis

\section{Introduction}

Trichomonas vaginalis (T. vaginlis) is the most prevalent sexually transmitted pathogen accounting for 180 million infections annually (Weger et al, 2018). It is one of the most important cofactors increasing HIV transmission and giving adverse birth outcomes. In Saudi Arabia, Trichomonaisis is one of the most commonly STIs reported to the ministry of health during 2005 to 2012 with overall incidence of $18.4 \%$ of all STIs and average annual incidence of $9.1 \%$ (Memish et al, 2016). T. vaginalis infection is not only restricted to the vagina but the parasite may be found and isolated from all genitourinary structures. The classical symptoms associated with trichomoniasis include a yellowish-green odorous vaginal discharge; pruritus, dysuria, and "strawberry" cervix which is characterized by punctuate hemorrhagic lesions (Gary, 2005). T. vaginalis infect men as well as women with a comparable frequency, but in men symptoms are normally mild and infections are cleared by the host's immune system within weeks. In women, trichomonaisis could persist for many years, and symptoms can attain a severity which is debilitating (Swygard $e t$ $a l, 2004)$. But, asymptomatic disease exists in both men and women (Leitsch, 2016).
Accurate diagnosis for trichomonasis is a fundamental issue for successful treatment that helps controlling its spread. Diagnosis cannot be made entirely on the basis of symptoms and clinical approach as it may mimic those of other STDs. So, laboratory based diagnosis has to be considered necessary for early and accurate diagnosis (Petrin et al, 1998; Garber, 2005). Detection of $T$. vaginalis was performed by several laboratory methods that varied in sensitivity and specificity (Radonjic et al, 2006; Memish et $a l, 2016)$. Culture is still considered the "gold standard" for diagnosis of trichomoniasis. However, it has many disadvantages, as it is costly, laborious and time-consuming as up to 7 days are needed for identification of positive cultures (Nye et al, 2009). The wet mount preparation of vaginal discharge was the commonest method for diagnosis. However, examination of specimens must be performed within $30 \mathrm{~min}$ after collection to enable visualization of viable and motile protozoa; a limitation that may affect its sensitivity (Radonjic et al, 2006). In set up lacking immediate microscopic facilities, staining techniques like Giemsa and Gram staining are very useful where prepared and fixed smears can be transported to laboratory for staining and diagnosis. With large number 
of patients attending gynecological outpatient clinics an immediate examination of vaginal swab samples is virtually impossible. Unlike wet mount examination, delay in transport has no significant impact on staining methods reliability for diagnosis.

The present study aimed at detection of $T$. vaginalis in vaginal swab specimens from clinically suspected women in Jazan, KSA, using wet mount examination, Giemsa stain, Gram stain and culture, and evaluation of the performance of staining techniques in diagnosis of $T$. vaginalis compared to culture as the reference method.

\section{Subjects and Methods}

The study subjects were women in Jazan City who had clinical manifestations suggestive of trichomoniasis. Each woman was asked to fill a questionnaire covering the personal data, clinical symptoms, marital status and obstetric and gynecological history. Vaginal swab samples were obtained from women fulfilling the following inclusion and exclusion criteria using sterile vaginal swabs provided to the patients.

Inclusion criteria: a- Married female $\geq 18$ years old, b- Complaining of one or more trichomoniasis symptoms (yellowish or green color vaginal discharge with or without itching, foul smell vaginal discharge, lower abdominal colic, pain in urination, pain in intercourse).

Exclusion criteria: a- Unmarried female, b- Pregnant female, c- During menstrual period, and d- During puerperal period.

Vaginal secretions were collected from the available responders to participate in the study who attend the gynecology outpatient clinics at different health centers in Jazan. Patients` self-obtained vaginal swabs were collected (Crucitti et al, 2003; Van Der Pol et al, 2014). The patient was informed to insert a single swab into the vagina and to rotate the swab three times. Each swab was suspended in $2 \mathrm{ml}$ of $0.85 \%$ normal saline supplemented with two drops of $5 \%$ glucose. Each sample was labeled and quickly transported to the Parasitology Laboratory,
Faculty of Applied Medical Sciences, Jazan University, for complete testing within 30 minutes. Before testing, the swabs were vigorously shaken to displace all the parasites into the saline solution.

Procedures: Each vaginal specimen was subjected to the following:

Wet mount Examination: A drop of saline with suspended vaginal secretions was placed on a $22 \times 40$ glass slide and covered with a cover slip. The preparation was examined microscopically for motile $T$. vaginalis under $\times 10 \& \times 40$ objectives. The vaginal secretion was characterized by the presence of the squamous epithelial cells while the trichomonads were identified by their size $(10-20 \mu \mathrm{m})$, round or oval shape, and characteristic quivering or twitching motility (Mahmoud et al, 2015).

Giemsa staining: A drop of saline with vaginal secretions was smeared on a $22 \times 40$ glass slide and allowed to air- dry then fixed by immersion in methanol for one minute. The slides were stained with stock Giemsa stained diluted 1:9 with phosphate buffer solution $(\mathrm{pH} \mathrm{7.2)}$ for $10 \mathrm{~min}$. Slides were washed with running faucet water, air dried and scanned for parasites at $10 \times 100$ magnification. No less than 30 fields were inspected before a negative finding was recorded. Trophozoite nucleus is round or oval, the flagella and undulating membrane appeared (Mahmoud et al, 2015).

Gram staining: Vaginal smear was prepared and fixed as for Giemsa staining then subjected to gram staining method as follows: the slide was flooded with crystal violet for one minute, the dye was poured off and the slide was delicately washed in tap water and depleted against a paper towel. The smear was presented to Gram's iodine for one minute by washing with iodine, at that point including more iodine and leaving it on the smear until the minute is over, then, the smear was washed with faucet water and drained. The slide was washed with $95 \%$ ethanol, then, washed with tap water at the end of the 30 seconds to stop the decoloriza- 
tion. The smear was stained with $0.25 \%$ safranin for 30 seconds, washed, drained, blotted, and examined by microscope. T. vaginalis trophozoites appeared as pear shape with a round or oval nucleus, flagella and undulating membrane \& axostyle appeared, and cytoplasm might be lacked (Harrington and Williams, 1999).

Modified thioglycolate culture: The medium was used at a concentration of $29.5 \mathrm{~g}$ in every liter of distilled water. The well mixed medium was dispensed in $10 \mathrm{ml}$ amounts in glass tubes fitted with screw-caps and sterilized by autoclaving (with caps loosened) at $121^{\circ} \mathrm{C}$ for 15 minutes. When cool, the tubes caps were tightened and each tube top was covered with a foil cap and stored in refrigerator. Just before use, the media were taken out of refrigerator and let to take the room temperature, and then, horse serum, Amphotericin B, Penicillin G, and Gentamicin were added (Pouch et al, 1996). Culture tubes were inoculated with the vaginal swab samples and incubated at $37^{\circ} \mathrm{C}$. The culture was microscopically examined for trophozoites after $24 \mathrm{hr}$ by taking a drop of medium from the base of each tube. This was done at two days and preceded up to seventh day before negative culture was disposed of.

Statistical analysis: For evaluation of diagnostic techniques, sensitivity, specificity, PPV \& NPV were ascertained for each test contrasted with culture. Chi-square test was used. A significant difference was considered when $\mathrm{p}<0.05$. Kappa test was used to identify agreement between diagnostic tests.

Ethical considerations: Patients were notified about the aim of study and informative written consent was obtained prior to participation. Confidentiality of the patients was maintained through the duration of the research. Each patient was informed with the test result.

\section{Results}

The present study included 40 female patients suffering from symptoms suggestive of trichomoniasis, with ages ranged from 18-52 years. Most of them were in group 1835 years $(27 / 40,67.5 \%)$. The commonest clinical symptoms were vaginal discharge $(17 / 40,42.5 \%)$ and vaginal discharge and itching $(13 / 40,32.5 \%)$ while dysuria was reported in 10 out of 40 subjects $(25 \%)$.

T. vaginalis was identified in 11 out of 40 samples by culture method $(27.5 \%), 10$ samples by wet mount (25\%), 8 samples by Gram stain (20\%) and 7 samples by Giemsa staining $(17.5 \%)$. When the results of staining methods were combined together the number of positive samples increased to 10 samples (25\%) (Tab. 1; Fig. 1). Compared to culture as standard method, Giemsa stain was more sensitive and specific than both wet mount and Gram stain. Giemsa stain showed $63.6 \%$ sensitivity compared to 54.5 $\%$ sensitivity for wet mount or Gram staining methods. Giemsa stain specificity was $100 \%$ compared to $86.2 \%$ for wet mount and $93.1 \%$ for Gram staining. Besides, a substantial agreement was found between culture and Giemsa staining $(\mathrm{Kappa}=0.717)$ while moderate agreements of wet mount $($ Kappa $=0.419)$ and Gram staining (Kappa $=$ 0.52 ) with culture were observed (Tabs. $2 \&$ 3 ). Discrepant outcomes were seen in five samples using the different diagnostic methods in the present work. The 5 samples were negative by culture, 3 of them were positive by wet mount \& 2 were positive by wet mount and Gram stain. So, only 35 samples were included in analysis of $T$. vaginalis infection among different age groups and different clinical manifestations. Trichomoniasis was more common among patients in age group 36-45 years compared to other age groups (Tab. 4) but without significant difference $(p>0.05)$. T. vaginalis in-fection was more commonly presented with vaginal discharge and itching, followed by vaginal discharge only and dysuria but with-out significant difference (Tab. 5). 
Table 1: Comparison of different methods for detection of T. vaginalis $(\mathrm{n}=40)$.

\begin{tabular}{|l|c|c|}
\hline Diagnostic method & Positive No. (\%) & Negative No. (\%) \\
\hline Culture & $11(27.5 \%)$ & $29(72.5 \%)$ \\
Wet mount & $10(25 \%)$ & $30(75 \%)$ \\
Gram stain & $8(20 \%)$ & $32(80 \%)$ \\
Giemsa stain & $7(17.5 \%)$ & $33(82.5 \%)$ \\
Gram stain and Giemsa stain & $10(25 \%)$ & $30(75 \%)$ \\
\hline
\end{tabular}

Table 2: Wet mount examination and staining methods for T. vaginalis compared to culture.

\begin{tabular}{|c|c|c|c|c|}
\hline \multirow{2}{*}{ Test } & \multicolumn{2}{|c|}{ Culture } & \multirow{2}{*}{ Kappa value } & \multirow[b]{2}{*}{ Statistical analysis } \\
\hline & Positive $(n=11)$ & Negative $(n=29)$ & & \\
\hline $\begin{array}{l}\text { Wet mount: } \\
\text { Positive (10) } \\
\text { Negative (30) }\end{array}$ & $\begin{array}{l}6(54.5 \%) \\
5(45.5 \%)\end{array}$ & $\begin{array}{c}4(13.8 \%) \\
25(86.2 \%)\end{array}$ & $\begin{array}{c}0.419 \\
\text { (moderate } \\
\text { agreement) }\end{array}$ & $\begin{array}{l}P \text { value } \\
<0.001\end{array}$ \\
\hline $\begin{array}{l}\text { Gram stain: } \\
\text { Positive (8) } \\
\text { Negative (32) }\end{array}$ & $\begin{array}{l}6(54.5 \%) \\
5(45.5 \%) \\
\end{array}$ & $\begin{array}{c}2(6.9 \%) \\
27(93.1 \%) \\
\end{array}$ & $\begin{array}{c}0.520 \\
\text { (moderate } \\
\text { agreement) }\end{array}$ & $\begin{array}{l}P \text { value } \\
<0.001 \\
\end{array}$ \\
\hline $\begin{array}{l}\text { Giemsa stain: } \\
\text { Positive (7) } \\
\text { Negative (33) }\end{array}$ & $\begin{array}{l}7(63.6 \%) \\
4(36.4 \%)\end{array}$ & $\begin{array}{c}0 \\
29(100 \%)\end{array}$ & $\begin{array}{c}0.717 \\
\text { (substantial } \\
\text { agreement) }\end{array}$ & $\begin{array}{l}P \text { value } \\
<0.001\end{array}$ \\
\hline
\end{tabular}

Significant at $p<0.05$

Table 3: Efficacy of different tests compared to culture.

\begin{tabular}{|l|c|c|c|c|}
\hline Test & Sensitivity & Specificity & PPV & NPV \\
\hline Wet mount & $54.5 \%$ & $86.2 \%$ & $60 \%$ & $83.3 \%$ \\
\hline Gram stain & $54.5 \%$ & $93.1 \%$ & $75 \%$ & $84.4 \%$ \\
\hline Giemsa stain & $63.6 \%$ & $100 \%$ & $100 \%$ & $87.9 \%$ \\
\hline
\end{tabular}

PPV: positive predictive value, NPV: negative predictive value

Table 4: Relationship of T. vaginalis positive cases (by culture) and age groups.

\begin{tabular}{|l|l|l|}
\hline Age group (years) & Number of cases & Number of positive cases $(\%)$ \\
\hline $18-25$ & 12 & $4(33.3 \%)$ \\
\hline $26-35$ & 12 & $3(25 \%)$ \\
\hline $36-45$ & 7 & $3(42.8 \%)$ \\
\hline $46-52$ & 4 & $1(25 \%)$ \\
\hline Total & 35 & $11(31.4 \%)$ \\
\hline$P$-value & 0.861 (not significant) \\
\hline
\end{tabular}

Table 5: Relationship of $T$. vaginalis infection and t clinical manifestations.

\begin{tabular}{|l|c|c|}
\hline Clinical symptom & No. of cases & No. of T. vaginalis positive (\%) \\
\hline Vaginal discharge & 15 & $4(36.4 \%)$ \\
\hline Vaginal discharge and itching & 11 & $6(54.5 \%)$ \\
\hline Dysuria & 9 & $1(9.1 \%)$ \\
\hline$P-$ value & \multicolumn{2}{|c|}{0.099 (not significant) } \\
\hline
\end{tabular}

\section{Discussion}

T. vaginalis is the second most commonly sexually transmitted infection in Saudi Arabia between 2005 \& 2012 (Memish et al, 2016). In the present study, 40 patients with clinical manifestations suggestive of T. vaginalis infection were examined using 4 methods. Sensitivity and specificity were calculated for wet mount, Gram stain and Giemsa stain methods using the culture as standard method for comparison. Sensitivity of wet mount examination was $54.5 \%$ \& specificity was $86.2 \%$. Sensitivity of Gram`s stain was $54.5 \%$ \& specificity was $93.1 \%$ while Giemsa stain sensitivity was $63.6 \%$ \& specificity was $100 \%$. In the present study, low sensitivity of wet mount $(54.5 \%)$ agreed with others (Khatoon et al, 2014; Mahmoud et al, 2015; Menezes, et al, 2016). They reported sensitivity values of $33.3 \%$ to $67.6 \%$. This might be due to deterioration of trichomonads to lose motility, retract flagella, becoming rounded and difficult to distinguish from other structures, such as leucocytes or nuclei 
of vaginal epithelial cells. Khatoon et al. (2014) explained the low sensitivity of wet mount by presence of less number of organisms that led to negative finding.

In the present study, Gram stain results were equivalent to that of wet mount method. This finding agreed with Angelika et al. (2002) who reported that Gram stain was substituted for other diagnostic techniques if immediate microscopy was impracticable Detection rate of trichomonads could be increased if one was aware of recognizing them in Gram stain smears (Sobrepena, 1980). But, five positive samples by culture were not recognized by Gram stain, which could indicate that trichomonads were more like polymorph nuclear leukocytes.

In the present study, Geimsa stain gave sensitivity of $63.6 \%$, which agreed with 41$100 \%$ sensitivity reported (Fernando et al, 2011; Khatoon et al, 2014; Paliwal et al, 2017). The inability of Giemsa stain to detect the trophozoites in 4/11 culture positive might be due to the fact that during stain processing, trophozoites were damaged or lost (Mahmoud et al, 2015).

In the present study, 5 samples were negative by culture but positive by using wet mount and Gram stain. This agreed with other studies (Crucitti et al, 2003; Patil et al, 2012) which proved that the sensitivity of culture in liquid media, such as Diamonds and Trichosel was 85 to $95 \%$. Regardless of that culture technique proved excellent for visualization of trichomonads, yet, it was not used in routine laboratories. It is timeconsuming required 7 days for growth. During which patient was on spreading disease before treatment (Mahmoud et al, 2015).

\section{Conclusion}

Trichomoniasis is usually diagnosed clinically, staining techniques used as supportive diagnostic methods in lack of microscopic facility. T. vaginalis diagnosis using staining methods would not be affected by delay in transportation, which decreases morbidity and adverse outcomes of trichomoniasis.

\section{References}

Weger, BO, Klein, DA, Bowsher, BL, Weir, L F, Roberts, TA, 2018: Measuring Community Prevalence of Trichomonas vaginalis infection to guide local screening practices: A process improvement project. Sex. Transm. Dis. Sep 13. doi: 10.1097/OLQ.0000000000000913

Angelika, S, Angelika, K, Lilianna, T, 2002: Detection of Trichomonas vaginalis on Modified Columbia Agar in the Routine Laboratory. J. Clin. Microbiol. 40:3277-80.

Crucitti, T, Van Dyck, E, Tehe, A, Abdellati, S, Vuylsteke, B, et al, 2003: Comparison of culture and different PCR assays for detection of Trichomonas vaginalis in self-collected vaginal swab specimens. Sex. Transm. Infect. 79:393-8.

Fernando, SD, Herath, S, Rodrigo, C, Rajapakse, S, 2011: Improving diagnosis of Trichomonas vaginalis infection in resource limited health care settings in Sri Lanka. J. Glob. Infect. Dis. 3, 4:324-8.

Garber, GE, 2005: The laboratory diagnosis of Trichomonas vaginalis. Can. J. Infect. Dis. Med. 16, 1:35-8.

Gary EG, 2005: The laboratory diagnosis of Trichomonas vaginalis. J. Infect. Dis. Med. Microbiol. 16, 1:35-8.

Harrington, B, Williams, D, 1999: Recognizing trichomonads in gram-stained smears. Lab. Med. 30, 12:803-7.

Khatoon, R, Jahan, N, Khan, H, Rabbani, T, Ahmad, S, 2014: Evaluation of different staining techniques in the diagnosis of Trichomonas vaginalis infection in females of reproductive age group. J. Clin. Diag. Res. 8, 12:DC05-8.

Leitsch, D, 2016: Recent Advances in the Trichomonas vaginalis field. F1000Res. 5:162-4. Mahmoud, A, Sherif, N, Abdella, R, El-Genedy, A, El Kateb, A, et al, 2015: Prevalence of Trichomonas vaginalis infection among Egyptian women using culture and Latex agglutination: cross-sectional study. BMC Women's Hlth. 15:7-11.

Menezes, C, Mwllom, M, Tasca, T, 2016: Comparison of permanent staining methods for the laboratory diagnosis of trichomoniasis. Rev. Inst. Med. Trop. Sao Paulo 58:5-8.

Memish, ZA, Filemban, SM, Al-Hakeem, R F, Hassan, MH, Al-Tawfiq, JA, 2016: Sexually trans-mitted infections case notification rates in the Kingdom of Saudi Arabia 2005-2012. J. Infect. Dev. Ctries 10, 8:884-7. 
Nye, MB, Schwebke, JR, Body, BA, 2009: Comparison of APTIMA Trichomonas vaginalis transcription-mediated amplification to wet mount microscopy, culture, and PCR for diagnosis of trichomoniasis in men and women. Am. J. Obstet. Gynecol. 200, 2:e188-97.

Paliwal , V, Jain, A, Laghawe, A, Navinchandra, K, Prabhu, T, 2017: Comparison of wet mount examination with Giemsa staining and fluorescent staining for detection of Trichomonas vaginalis in clinically suspected cases of vulvovaginitis. Int. J. Curr. Microbiol. App. Sci. 6, 3:718-24.

Patil, MJ, Nagamoti, JM, Metgud, SC, 2012: Diagnosis of Trichomonas vaginalis from vaginal specimens by wet mount microscopy, in pouch $T v$ culture system, and PCR. J. Glob. Infect. Dis. 4, 1:22-5.

Petrin, D, Delgaty, K, Bhatt, R, Garber, G, 1998: Clinical and microbiological aspects of
Trichomonas vaginalis. J. Clin. Microbiol. Rev. 1:300-17.

Radonjic, IV, Dzamic, AM, Mitrovic, SM, ArsicArsenijevic, VS, Popadic, DM, et al, 2006: Diagnosis of Trichomonas vaginalis infection: The sensitivities and specificities of microscopy, culture and PCR assay. Eur. J. Obstet. Gynecol. Reprod. Biol. 126, 1:116-20.

Sobrepena, RL, 1980: Identification of Trichomonas vaginalis in Gram-stained smears. Lab. Med. 11, 8:558-60.

Swygard, H, Sena, AC, Hobbs, MM, Cohen, MS, 2004: Trichomoniasis clinical manifestations, diagnosis and management. Sex. Transm. Infect. 80:91-5.

Van Der Pol, B, Williams, JA, Taylor, SN, Catherine, L, Cammarata, CL, et al, 2014: Detection of Trichomonas vaginalis DNA by use of self-obtained vaginal swabs with the BD ProbeTec Qx Assay on the BD viper system. J. Clin. Microbiol. 52, 3:885-9

\section{Explanation of figure}

Fig. 1: Vaginal secretion samples. T. vaginalis trophozoites (Red arrow), Squamous epithelial cells (Black arrow). A \& B: wet mount smears. C\& D: Giemsa stained smears. E \& F: Gram stained smears

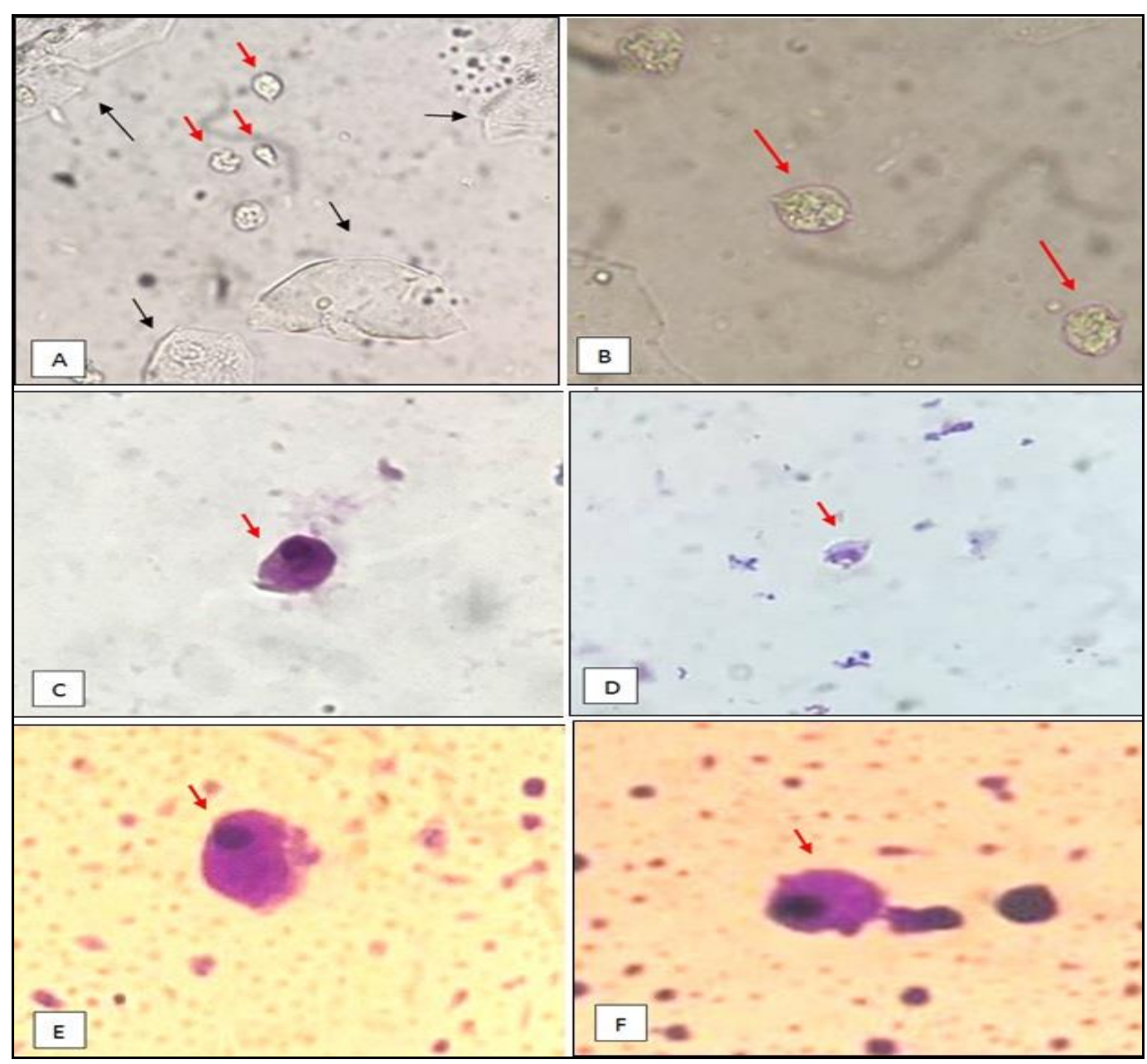

